\title{
Ab Initio Analysis of a Quantum Dot Induced by a Local External Potential in a Semiconducting Carbon Nanotube
}

\author{
T. KOSTYRKO ${ }^{a}$ And S. KROMPIEWSKI ${ }^{b}$ \\ ${ }^{a}$ Faculty of Physics, Adam Mickiewicz University \\ Umultowska 85, 61-614 Poznań, Poland \\ ${ }^{b}$ Institute of Molecular Physics, Polish Academy of Sciences \\ M. Smoluchowskiego 17, 60-179 Poznań, Poland
}

\begin{abstract}
Using the density functional theory we study the influence of external charge probes on the electronic structure of semiconducting carbon nanotubes in the vicinity of the Fermi level. We show that the spatially limited potential due to the probe can create localized electronic states in the energy gap and at the edges of the conductance band. By filling these localized states with additional electrons one obtains a quantum dot, which can be tuned by modifying the properties of the external charge probe. We analyze dependence of the electronic structure of the dot on the spatial extension of the potential as well as on the nanotube radius.
\end{abstract}

PACS numbers: 61.48.De, 85.35.Kt, 73.21.La, 73.22.-f

\section{Introduction}

Electronic properties of carbon nanotubes (CNTs; see Ref. [1] for a review) continue to attract much interest due to promises of their applications as replacements of standard Si-based devices in future molecular electronics. Numerous experimental efforts were devoted to fabrication and study of CNT-based quantum dots (CNTQD, see Ref. [2]), which were shown to work as single-electron transistors [3] or diodes. In many of these experiments metallic CNTs weakly coupled to the leads were used, where they showed characteristic features of the Coulomb blockade (CB). In these devices the existence of the $\mathrm{CB}$ peaks is limited to low temperature region because of relatively small values of the Coulomb charging energies, which depend inversely proportional on the CNT size.

In order to extend the operational capabilities of CNTs to room temperatures a radical decrease in size of the active CNT part is necessary. This can be achieved by separating out a small part of a metallic CNT from the rest of it by barriers created by strong defects [4, 5] resulting from pressing the CNT by atomic force microscope (AFM) tip. The charge occupation and transport properties of such CNTQDs can be then controlled with a charged AFM tip, working as a gate electrode [6].

In the present paper we consider a possibility of creating CNTQD in semiconducting CNTs of finite length, by application of a strongly local static Coulomb potential. In a practical application, this potential could be created by a system of metallic CNTs, alternately charged and aligned transversely to the semiconducting CNT. The potential well created in the semiconducting CNT near the potential minimum, can provide a system of local electronic levels being able to accommodate extra electrons. The depth, the size of the well and the separation of the electronic levels could be controlled with the external Coulomb potential and in this way one can tune the CNTQD to the desired working conditions. The resulting trap could be populated with electrons by means of a source-drain voltage pulse, or by exciting electrons to the conducting band by absorption of quanta of electromagnetic field. According to the needs such a device could work as a memory cell, a transducer or a single electron transistor.

Here we study a simple model of such a tunable CNTQD by means of $a b$ initio computations. We consider a model of the tunable CNTQD composed of a system of semiconducting CNT and two closed shell ions, mimicking the external potential probe. We investigate properties of this model of CNTQD as a function of spatial extension of the external potential and the CNT diameter. The magnetic structure of the double occupied CNTQD is discussed in the light of a possibility of using a magnetic field to control occupation of the dot.

\section{Numerical results and discussion}

In our computation we use the density functional theory based (DFT-based) SIESTA package [7]. The package applies the pseudopotential method and finite range basis functions allowing one to deal with structures of hundreds of atoms even on an average computer. We applied periodic boundary conditions along the nanotube axis to neglect the in-gap states which are localized at 
the ends of finite size CNTs. The largest CNT we studied: (14,0) with 30 unit cells, included $1680 \mathrm{C}$ atoms. To limit the computer memory usage (8 GB), we used the SZ (single-zeta [7]) basis with one orbital per each valence state $(2 s, 2 p)$ of the $\mathrm{C}$ atom and we reduced the size of the wave functions with pseudoatomic orbital $(\mathrm{PAO})$. Energy shift $=0.02$ Ry [7]. All our calculations are based on the Troullier and Martins pseudopotential [8] and local density approximation (LDA) in the Perdew and Zunger parameterization [9]. Two external point charges were obtained from two $\mathrm{K}$ atoms with spatially reduced atomic basis. Because the atomic levels of such prepared $\mathrm{K}$ atoms were much higher than the Fermi level of the CNT, the two $s$ electrons of $\mathrm{K}$ atoms were transferred to the CNT in the course of self-consistent-field calculations. We note that our system composed of the CNT and two (ionized) K atoms is still neutral, although it can possess a sizeable dipole moment.

The characteristic feature of the spectrum of CNT is double degeneracy of the orbital energy levels, related to two energetically equivalent modes of the electron motion along the tube, accompanied by either clockwise or anticlockwise rotation around the tube [10]. The degeneracy can also be seen in Fig. 1, where the Kohn-Sham (KS) spectrum for a semiconducting zigzag $(14,0)$ CNT is shown.

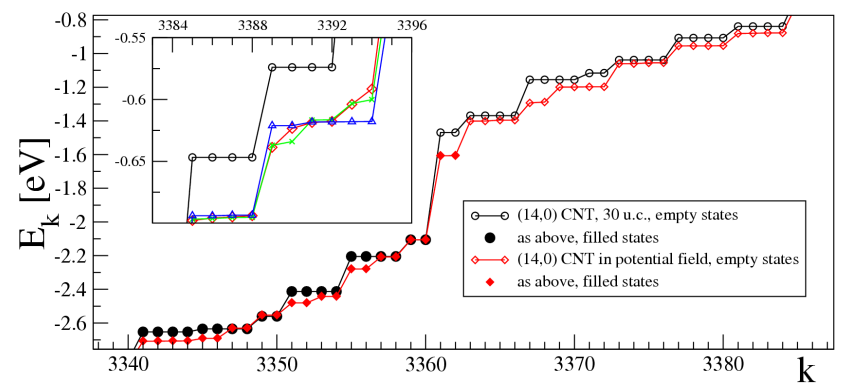

Fig. 1. The single particle KS energy spectrum of $(14,0) \mathrm{CNT}, 30$ unit cells (u.c.) (integer $k$ numbers the mode). Circles: neutral CNT. Diamonds: CNT charged with 2 electrons in an electrostatic field created by a system of 2 point $+|e|$ charges. Inset: part of the spectrum showing dependence on the position and the orientation of the system of charges with respect to CNT: external with parallel alignment to CNT - diamonds, external with transverse alignment to the CNT - crosses, internal at the CNT axis - triangles. In all the cases the distance of charges to the CNT surface was about $4 \AA$, and separation of the charges was $d=6 \AA$. All the plots for the charged CNT were shifted to match the HOMO level of the neutral CNT $(k=3360)$ for the ease of comparison.

Double charging of the CNT with a pair of electrons and putting it under the influence of the external local field produced by a system of two positive charges, noticeably perturbs the electronic spectrum at the edge of the conductance band, dragging a pair of electron levels by a fraction of $\mathrm{eV}(\approx 0.15 \mathrm{eV})$ inside the gap. One can note that the nearly double degeneracy of the orbital states is still present under the influence of the external local potential. This conclusion is approximately independent of the orientation of the system of the two positive charges with respect to CNT axis. Only at the distance of about $1 \mathrm{eV}$ from the Fermi level the effects of a symmetry breaking external potential on the spectrum can be readily noticed (see inset in Fig. 1).

The new highly occupied molecular orbital (HOMO) level of the double charged CNT, unlike the HOMO level of the neutral CNT, is localized near the bottom of the potential well due to the presence of the positive charges (see Fig. 2). Let us note that unlike the metallic nanotubes, where the quasibound states are formed near the Fermi level under the influence of a local perturbation, the truly localized HOMO state is much more spatially confined in the present case. Due to the spatial limitation of HOMO the Coulomb interactions between two electrons in the HOMO level may be much higher than between extra electrons in the perfect CNT. In this case the electrons can take advantage of the double degeneracy of the HOMO level and occupy the different orbital states to minimize on-site Coulomb repulsion, producing in this way a triplet state rather than a closed shell singlet one. The triplet states were indeed observed in semiconducting CNTs in magnetic field [10]. In order to verify this possibility in the present case we compared the energy of closed shell solution with spin polarized one with fixed value of the total spin $S=1$. For the $(14,0)$ CNT with 30 u.c. we found that the energy of the singlet solution was smaller by a few meV than that of the triplet one.

The results for the $(8,0)$ CNT are presented in Fig. 3. We found that the electronic structure of the neutral $(8,0)$ CNT near the Fermi level depends on its length. The lowly unoccupied molecular orbital (LUMO) level of the neutral $(8,0)$ CNT with 30 u.c., as well as the HOMO level of the double charged CNT are not degenerate, in contrast to those in the $(14,0)$ CNT. This is probably a result of interplay of $\sigma-\pi$ interactions and finite size effects of the CNT. Indeed, in the CNT $(8,0)$ with 50 u.c. the corresponding states were doubly degenerate as the ones in $(14,0)$ CNT with 30 unit cells. By comparing the results for the shorter $(8,0) \mathrm{CNT}$ and the longer one we can evaluate importance of the degeneracy of the orbital levels for the stability of the triplet solution over the singlet one. In Fig. 3 the dependence of difference between the energy of the triplet and singlet solution, $\Delta_{\mathrm{ST}}$, is plotted as a function of the distance $d$ between the charges aligned in parallel to the CNT axis. For the CNT with 30 u.c. and the charges put outside the CNT, $\Delta_{\mathrm{ST}}$ reaches a value of $\approx 0.15 \mathrm{eV}$ for $d=3 \AA$ and decreases with increase in $d$, slowly approaching the limit of two independent non-interacting spins. By putting the charges right at the CNT axis, i.e. in the axially symmetric position, we reduce the value $\Delta_{\mathrm{ST}}$ by about a factor of 10 . For the $(8,0) \mathrm{CNT}$ with 50 u.c. we finally find that the triplet solution has lower energy than the singlet one. 


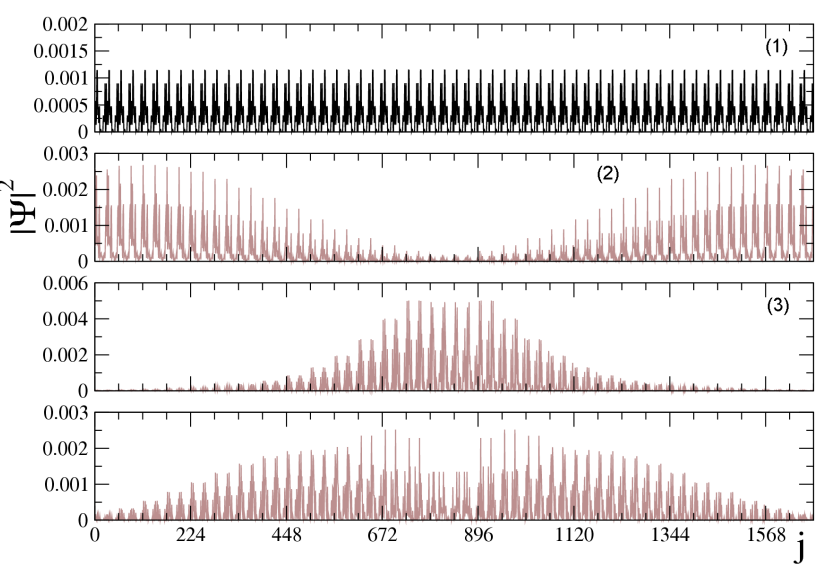

Fig. 2. The squared coefficients of the extent of the KS wave function in pseudoatomic orbitals (PAO, the dominating $2 p_{x}$ components are only shown) corresponding to the CNT as described in Fig. 1 for the neutral CNT (top figure) and the double electron charged CNT in the field of two positive elementary charges - see diamonds in Fig. 1 (all the other figures). From top to the bottom: (1) HOMO orbital of the neutral CNT, (2) occupied level No. 3360 of double electron charged CNT, (3) HOMO orbital (No. 3361) of the double charged CNT, (4) LUMO orbital of the double charged CNT (No. 3363). The integer $j$ is the $\mathrm{C}$ atom index.

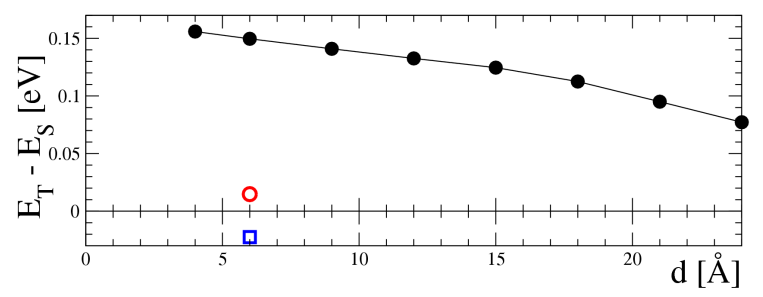

Fig. 3. Energy difference between the spin polarized (triplet) solution and the closed shell singlet for the double charged $(8,0) \mathrm{CNT}$ in the field of two positive charges aligned parallel to the CNT, as a function of distance between the charges. Filled circles: CNT with 30 unit cells, charges placed outside the CNT, $4 \AA$ away from its surface. Open circle: as above, but for charges inside the CNT at its axis. Square: for the CNT $(8,0)$ with 50 unit cells, charges are positioned at the CNT axis.
In conclusion, our results show that it should be possible in experiment to use a local charge probe to create and modify the quantum dot in the defect-free semiconducting CNT. Concerning the relative stability of the triplet and singlet solutions, they can probably be modified by using a better function basis (DZP rather than $\mathrm{SZ}$ ) or more exact DFT functionals (i.e. B3LYP instead of LDA). However they suggest that the degeneracy of orbital levels stabilizes the triplet solution more effectively in narrow CNTs rather than in the wider ones.

\section{Acknowledgments}

The work was supported as a part of the European Science Foundation EUROCORES Programme FoNE by funds from the Ministry of Science and Higher Education and EC 6FP (contract No. ERAS-CT-2003-980409). S.K. thanks also the EU grant CARDEQ, contract No. IST-021285-2.

\section{References}

[1] J.C. Charlier, X. Blase, S. Roche, Rev. Mod. Phys. 79, 677 (2007).

[2] S. Sapmaz, P. Jarillo-Herrero, L.P. Kouwenhoven, H.S.J. van der Zant, Semicond. Sci. Technol. 21, (20S52- S63)06.

[3] M. Bockrath, D.H. Cobden, P.L. McEuen, N.G. Chopra, A. Zettl, A. Thess, R.E. Smalley, Science 275, 1922 (1997).

[4] L. Chico, M.P. López Sancho, M.C. Muñoz, Phys. Rev. Lett. 81, 1278 (1998).

[5] H.W.Ch. Postma, T. Teepen, Z. Yao, M. Grifoni, C. Dekker, Science 293, 76 (2001).

[6] M. Bockrath, W. Liang, D. Bozovic, J.H. Hafner, C.M. Lieber, M. Tinkham, H. Park, Science 291, 283 (2001); M.T. Woodside, P.L. McEuen, Science 296, 1098 (2002).

[7] P. Ordejón, E. Artacho, J.M. Soler, Phys. Rev. B 53, R10441 (1996); J.M. Soler, E. Artacho, J.D. Gale, A. García, J. Junquera, P. Ordejón, D. Sánchez- Portal, J. Phys., Condens. Matter 14, 2745 (2002).

[8] N. Troullier, J.L. Martins, Phys. Rev. B 43, 1993 (1991).

[9] J.P. Perdew, A. Zunger, Phys. Rev. B 23, 5048 (1981).

[10] A. Makarovski, L. An, J. Liu, G. Finkelstein, Phys. Rev. B 74, 155431 (2006). 